\title{
Ueber die Bestimmung der Salpetersäure nach Schlösing;
}

\author{
von E. Reichardt, Prof. in Jena.*)
}

Die Vortheile der Schlösingschen Methode der Salpetersäurebestimmung sind so vielfach anerkannt worden, dass eine Wiederholung der empfehlenden Momente unnöthig sein dürfte; der allgemeinen Verwendung derselben steht jedoch namentlich der Gebrauch des Quecksilbers als Sperrflüssigkeit entgegen, da dies doch nur in eigentlichen chemischen Laboratorien in genügender Masse und passender Verwendbarkeit geboten wird. Um hier Aenderungen zu bewerkstelligen, wurde zunächst auf die ausführliche Arbeit von Schlösing zuriickgegangen, welche sich in Erdm. Journ. f. pr. Chemic 1854. Bd. 62. S. $142-169$ mitgetheilt findet.

Schlösing unterscheidet die Bestimmung sehr kleiner und grösserer Mengen Salpetersäure und ändert für ersten Zweck sogar Apparat wie Verfahren, indem er das erzeugte Stickoxydgas durch einen Strom reiner Kohlensäure austreiben lässt und als Absorptionsmittel nicht Kalkwasser, sondern concentrirte beisse Kalilauge gebraucht.

Bei der äusserst geringen Löslichkeit des Stickoxydes in Flüssigkeiten überhaupt, in Wasser und Alkali haltendem Wasser, war nicht anzunehmen, dass ein irgend erheblicher Fehler durch Umgehung des Quecksilbers als Sperrflüssigkeit eintrete, nur müsste man mit Kalkwasser oder verdünnter Natronlauge arbeiten, was abermals Unannehmlichkeiten mit sich führt.

Ganz nach Schlösing's Weise wurde 0,1 Grm. KO, $\mathrm{NO}^{5}$ mit $\mathrm{FeCl}$ und $\mathrm{ICCl}$ behandelt, nachdem durch Einkochen der wässerigen Salpeterlösung. in dem Zersetzungsgefässe letzteres luftleer gemacht worden war, und das entweichende, durch längeres Kochen ausgetriebene, Stickoxydgas unter Wasser in graduirter Röhre aufgefangen.

*) Als Separatabdruck aus Fresenius' Zeitschr. f. analyt. Chemie vom Hrn. Verfasser erhaiten.

Dio Red. 
I. $0,1 \mathrm{Grm}$. KO, $\mathrm{NO}^{5}$ gaben $24,2 \mathrm{CC}$. $\mathrm{NO}^{2}$ bei $756,75^{\mathrm{mm} \cdot} \mathrm{B}$. and $19^{\circ} \mathrm{C}$. T. $=21,94 \mathrm{CC}$. $\mathrm{NO}^{2}$ bei $0,760 \mathrm{M}$. B. und $0^{\circ} \mathrm{C}$. $=0,02947 \mathrm{Grm} . \mathrm{NO}^{2}=0,09933 \mathrm{Grm} . \mathrm{KO}, \mathrm{NO}^{5}$ oder 99,33 Proc. der angewendeten Menge.

II. $0,1 \mathrm{Grm} . \mathrm{KO}, \mathrm{NO}^{5}$ gaben $24 \mathrm{CC}$. $\mathrm{NO}^{2}$,bei $0,757 \mathrm{M}$. B. und $16^{\circ}$ C. T. $=22,11 \mathrm{CC}$. bei Norm. Dr. und T. $=$ $0,029702 \mathrm{Grm} . \mathrm{NO}^{2}=0,10011 \mathrm{Grm}$. KO, NO $\mathrm{NO}^{5}=100,11$ Proc.

Jedenfalls würde demgemäss das auf diese Weise unter Wasser aufgefangene Stickoxydgas eine vollständige, genaue Bestimmung der Salpetersäure ermöglichen. Das Sperrwásser war kalt verwendet worden. Diese Art der Bestimmung würde aber ejgentlich nur bei reinen salpetersauren Salzen oder sehr geeigneten Gemischen anwendbar sein; es sollte hier auch nur der Versuch geboten werden, die Genauigkeit der Abscheidung des Stickoxydgases zu controliren.

Hierauf construirte ich nachstehenden Apparat, welcher hoffentlich die Schlösing'sche Methode wesentlich erleichtert und den allgemeinen Gebrauch ermöglicht.

Fig. 1.

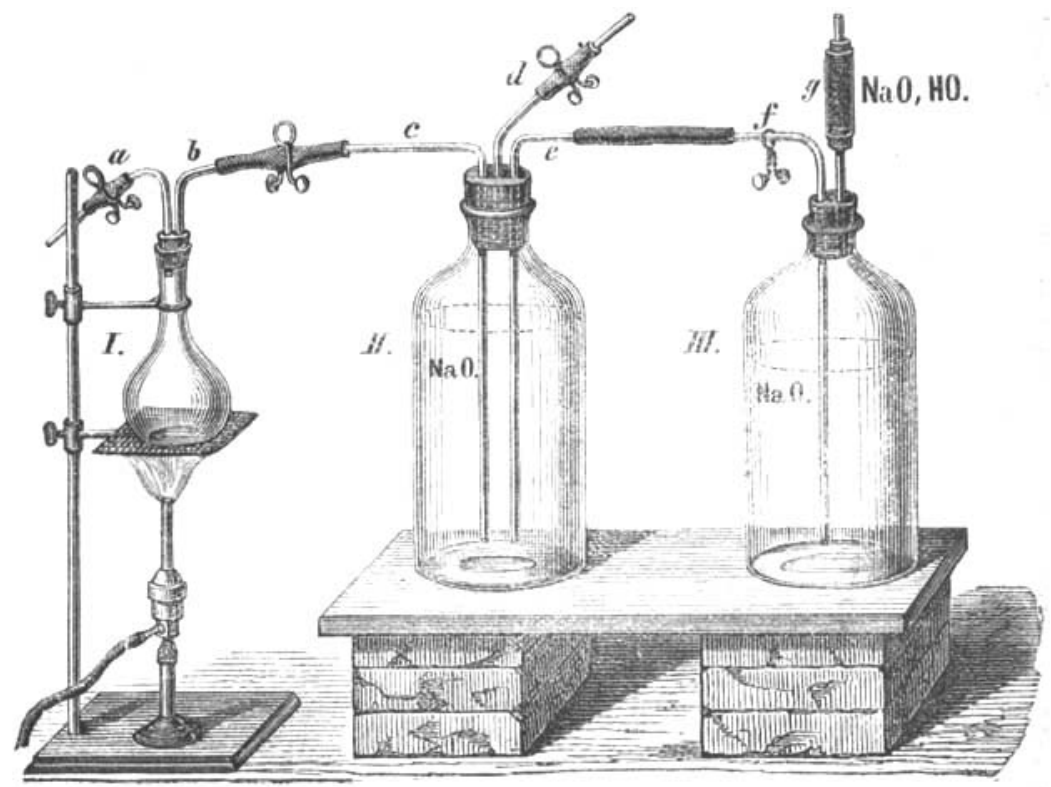


II. (Fig. 1) ist ein hohes, nicht zu breites Glasgefüss, welches mit dreifach durchbohrtem Kautschukstopfen verschlossen ist. Durch 2 Oeffnungen gehen die Röhren $\mathrm{c}$ und c, welche rechtwinkelig gebogen bis fast auf den Grund des Gefässes reichen. Röhre $d$ ist etwas aufgehend gebogen und schneidet genau mit der unteren Fläche des Stopfens ab.

III. ist eine grössere Flasche mit einer gleichfalls rechtwinkelig gebogenen Glasröhre f versehen, welche fast bis auf den Boden des Gefässes reicht und ferner mit $g$, einer passenden Röhre, gefüllt mit Stücken von Aetznatron, um einen Schutz nach Anssen żu gewähren. III. wird mit nicht zu verdünnter Natronlauge (circa $10 \mathrm{pC}$.) gefüllt, Röhre $f$ mit e durch ein Kautschukrohr verbunden; für etwa nöthigen Schluss findet sich bei f ein Quetschhahn vor.

Durch Einblasen bei g, oder Saugen bei d unter Schluss von c, treibt man die Lange nach Flasche II. über und füllt dieselbe vorsichtig vollstänig an, zuletzt schliesst man d und füllt die Röhre c noch durch Saugen an dem anliegenden Kautschukschlauche. Da es bci dem Verfahren nach Schlösing vor Allem darauf ankommt, alle Luft oder allen Sauerstoff zu entfernen, so bringt man jetzt bei c eine Wasserstoffentwickelung an, welche für' diese Zwecke stets vorräthig ist, etwa ähnlich einem Feuerzeuge nach Doebereiner; natiirlich muss das Wasserstoffgas selbst frei von Luft sein. Man leitet darauf Wasserstoffgas ein, während d geschlossen ist, wobei die Lauge ungehindert zwischen II. und III. circulirt. Hierauf schliesst man c und saugt bei d, am Geeignetsten mit einem Aspirator, die Lauge wieder auf, vermeidend, dass etwas Lauge mit in die aufsteigende Röhre gelange. In wenigen Minuten hat man die Operation $3-6 \mathrm{Mal}$ wiederholt und sich die Gewissheit verschafft, dass nur Wasserstoffgas die wenigen flüssigkeitsleeren Räume von II. und von den anschliessenden Röhren erfülle. Man schliesst nun die Quetschhähne bei $c$ und d (d hat an dem Kautschukrohr noch ein kleines Stückchen Glasrohr). Sobald wan geschlossen, spritzt man das offene Ende des Rohres d ganz voll Wasser und hindert so den Luftzutritt vollständig, kann auch jede weitere 
Verbindung ohne Luftzutrilt ermöglichen, sobald nur der äussere freie Schlauch und die Röhre völlig mit Wasser gefüllt sind.

Bei $\mathrm{c}$ ist dies derselbe Fall, es ist noch ohne Verbindung mit Fig. I. und wird sofort nach Abschluss der Wasserstoffleitung der nach Aussen gerichtete Theil des Kautschukrohres mit Wasser völlig gefüllt.

Diese Füllung des Apparates mit Lauge und Zuleitung des Wasserstoffgases ist bei einiger Uebung eine ungemein leichte Arbeit, und dient sodann der gefüllte Apparat zu oiner grossen Zahl Bestimmungen; nur muss beachtet werden, dass die Menge der Lauge nicht das Gefäss III. überfülle, was bei wiederholten Bestimmungen durch die allmähliche Vermehrung der Flüssigkeit geschieht. Man nimmt dann neue Lauge in der geeigneten Menge.

I. ist vollständig das Entwickelungsgefäss nach Schlösing, nur ersetzt, wie schon Grouven und Frühling angegeben und ich gleichfalls in meiner früheren Abhandlung hervorhob, durch Kochflasche mit Kautschukstopfen; jedoch glaube ich, noch eine wesentliche Verbesserung angebracht zu haben durch Anwendung eines doppelt durchbohrten Stopfens mit 2 Leitungsröhren, einer absteigenden, mit Kantschukrohr und einem kleinen Stück Glasrohr versehen a und einer ähnlichen oder etwas aufrecht gebogenen Röhre b zur Verbindung mit c. Sehr geeignet lässt man a etwas unter dem Stopfen hervorstehen.

Die betreffende, auf Salpetersäure zu prüfende Substanz wird in ziemlich concentrirtem, gelösten Zustande in I. gegeben und das Glas mit dem Stopfen verschlossen, beide Röh ren offen. Bei Wasser verdunste ich $1 / 2$ Liter, mit etwas Natron versetzt, bis fast zur Trockene und gebe die filtrirte Flüssigkeit in die Flasche I., mit Wasser nachspülend. Hierauf kocht man in der etwa 200 C.C. fassenden Flasche ein bis auf wenig Rückstand, um dadurch die Flasche völlig luftleer zu machen. Ist dies verrichtet, was in der Regel nur wenige Minuten beansprucht, so verbindet man sofort $b$ mit dem mit Wasser vollständig erfüllten äusseren Theile des 
Gummiscblauches von c, während durch a noch fortdauernd die Wasserdämpfe entweichen. Hierauf führt man a in eine concentrirte Lösung von Eisenchlorür und entfernt das Feuer. In ganz kurzer Zeit wird die Fisenchlorïrlösung eingesaugt. Man hüte sich jedoch, sie vollständig aufsaugen zu lassen, schliesse vielmehr vorher den Quetschhahn bei a, gebe etwas concentrirte Salzsäure vor und lasse von dieser ebenfalls einsaugen, um sowohl das Eisenchlorür vollständig in die Flasche zu bringen, als auch gleichzeitig die nothwendige Menge freier Salzsäure selbst. Man schliesse den Quetschhahn gleichfalls, bevor alle Säure aufgesaugt ist. Eine grosse Erleichterung und Verbesserung ist es jedoch, dass das später auftretende Stickoxydgas gar nicht durch die Röhre a, sondern durch $b$ geleitet wird.

Fresenius schreibt auf $0,2 \mathrm{Grm}$. NO $\mathrm{N}^{5}$ 1,5 Grm. Fe vor, d. h. eine mehr als doppelt genügende Menge, was den von Schlösing ermittelten Verbältnissen entspricht, da ein starkes Uebermaass von Eisenoxydullösung zur vollständigen Umsetzung nöthig ist. Fresenius titrirt das Eisen und schreibt dann natürlich eine genau zu wiegende Menge desselben vor. Für die hier vorliegenden Versuche genügt ein sehr starkes, annähernd bekanntes Uebermaass, wesshalb ich die concentrirte Eisenchlorïrlösung vorräthig halte und dann obigen Verhältnissen entsprechend davon verwende und circa gleichviel Salzsäure.

Hat man I mit der zu prüfenden, Salpetersänre haltenden Mischung versehen, sodann mit Eisenchlorür und Salzsäure, so giebt man sehr schwaches Feuer, annähernd 810 Minuten lang, dem Wasserbade entsprechend, und unter fortwährendem Schluss der Quetschhähne bei a und $b-c$; bemerkt man jedoch die Gasentwickelung durch Auftreiben des erst stark zusammengezogenen Schlauches bc, so öfnet man diesen, erhitzt stärker bis zum anhaltenden Kochen und treibt hierdurch das Stickoxydgas nach II über. Die mit übergehende Salzsäure wird sofort absorbirt, man sieht jedoch bald bleibendes Gas - Stickoxydgas - auftreten; die Flïssigkeit erwärmt sich bis zum Sieden, was jedenfalls die Absorp- 
tion von Gasen erschwert. Man unterbricht das Kochen in I, sobald keine bleibenden Gasblasen mehr auftreten, öffnet zu diesem Zwecke a, schliesst $b-c$ und entfernt die Flamme. Das von b zu entfernende Kautschukrohr b-c wird sofort mit Wasser vollgespritzt, um den Zutritt der Luft zu hindern.

Der weitere Verlauf ist wiederum ganz nach Schlös ing, mag jedoch der Vollständigkeit und etwas geänderten Sachlage wegen beschrieben werden.

Da die Natronlauge ungehindert von III nach II gelangen kann und umgekehrt, so findet hier ununterbrochen die nothwendige Ausgleichung statt und füllt sich Gefäss II sofort beim Erkalten wieder. Sogleich nach beendigter Gasentwickelung kocht man in einem etwa $200 \mathrm{CC}$. enthaltenden Glase, das mit durchbohrtem Gummistopfen und etwas aufgebogener Glasröhre nebst Gummischlauch und Quetschhahn versehen ist, etwas Wasser, um alle Luft auszutreiben; ist dies nach wenigen Minuten erlangt, so verbindet man augenblicklich den Kautschukschlauch mit dem oben vollständig mit Wasser angefüllten Röhrchen d. Beim Erkalten entsteht eine ziemlich starke Spannung, man öffnet den Quetschhahn bei d und saugt rasch alles Gas ans Gefäss II auf; die Lauge darf jedoch nicht in die Röhre gelangen. Gleichzeitig leitet man, mit bekannter Vorsicht hinsichtlich des Eintrittes von Luft, bei c wieder'etwas Wasserstoffgas ein, saugt dasselbe wieder bei d auf und wiederholt dies circa $3 \mathrm{Mal}$, um sicher alles Stickoxydgas in die Saugflasche genommen zu haben. Letztere schliesst man dann mit dem eigenen Quetschhahn, schliesst aber auch gleichzeitig c und d, spritzt die äusseren Oeffnungen voll Wasser und der Apparat ist zur folgenden Bestimmung wieder fertig u. s. w.

$\mathrm{Zu}$ dem in der Saugflasche befindlichen Stickoxydgas fügt man dann Sauerstoff zu, der stets noch von selbst aufgesaugt wird, bis keine Röthung mehr eintritt, lässt circa 25 Minuten ruhig stehen und titrirt hierauf die Säure.

Für diese Zwecke hält man sich natürlich Sauerstoff und Wasserstoff vorräthig, oder für letzteren eine sofort in Gang zu setzende Entwickelung. Abgesehen von dem Apparate 
ist der Gang vollständig der von Sohlösing angegebene. (Siehe Fresen. quantit. Analyse, 5. Aufl. 1863. S. 427.).

Zahlreiche Controlversuche mit Salpeterlösungen ergaben bei sehr sorgfältiger Arbeit genau 100 Proc., für gewöhnlich aber stets 96-98 Proc. der genommenen Menge wieder, eine Genauigkeit, welche sicher für die meisten Bestimmungen ausreichen diurfte.

Die von Fresenius eingeführte Modification dieser Bestimmung, das gebildete Eisenoxyd zu ermitteln, ist jedenfalls höchst genau, jedoch bei Anwesenheit leicht zersetzbarer' organischer Materien mit Uebelständen verknüpft, wesshalb dann immer wieder die Bestimmung des Stickoxydgases oder der daraus erzeugten Säure vorzuziehen wäre.

Ist der hier angegebene Apparat im Gange, so folgen dic Bestimmungen mit grosser Schnelligkeit, so dass eine Analyse kaum 1/2 Stunde Zeit beansprucht und ohne Unterbrechung fortgearbeitet werden kann.

\section{Zur Nachweisung der Salpetersäure.}

Von Demselben. ${ }^{*}$ )

Die Prüfung auf diese Säure oder auf salpetrige Säure, welche bei kleiner Menge gewöhnlich correspondirt, ist, wie die vielfach dahin einschlagenden Abhandlungen erweisen, von besonderer Wichtigkeit bei der Beurtheilung von Wasser als Trinkwasser oder für gesundheitspolizeiliche Zwecke und dessbalb der Mangel einer genauen Prüfungsmothode bei sehr grosser Verdünnung um so fühlbarer.

Unbestreitbar gewährt die qualitative und quantitative Bestimmung der Salpetersäure im Trinkwasser den sichersten Anhalt, äussere Einflüsse durch Infiltration oder Verwesung organischer Materien festzustellen.

Die zuletzt von Böttger besonders empfohlene Prüfung nach $\mathrm{Braun}{ }^{* *}$ ) mittelst schwefelsaurer Anilinlösung wurde

*) Als Separatabdruck aus Fresenius' Zeitschrift f. analyt. Chemie. IX. Jahrgang. 2. Heft, vom Hr. Verf. erhalten. II. $L$.

**) Fresenius' Zeitschrift 1867. S. 71. 